\title{
Cannabis and Inflammatory Mediators
}

\author{
Marcelo G. Lima ${ }^{a}$ Vitor S. Tardellia Elisa Brietzke ${ }^{b, c}$ Thiago M. Fidalgo ${ }^{a}$ \\ aDepartment of Psychiatry, Universidade Federal de São Paulo (Unifesp), São Paulo, Brazil; \\ bPos-Graduation Program in Pschiatry and Medical Psychology, Universidade Federal de São Paulo, \\ São Paulo, Brazil; 'Department of Psychiatry, Queen's University School of Medicine, Kingston, ON, Canada
}

\section{Keywords}

Cannabis · Cannabinoids · Inflammation · Inflammatory mediators

\begin{abstract}
Introduction: Although the recreational cannabis use is expressive worldwide, the literature about medical potential of cannabis extracts, including its anti-inflammatory properties, remains inconclusive. Methods: We screened all articles, published on the PubMed database, on inflammatory mediators and any information about cannabis use from 1980 to March 2019. Results: Six studies were included, and the main findings were as follows: (i) among healthy volunteers and cannabis users, cannabinoids seemed to decrease the inflammatory response, thus decreasing the immune response, which led to a higher risk of infections; (ii) among patients with multiple sclerosis, cannabinoids seemed to have little impact on the inflammatory markers' levels. Discussion: Although cannabis use can produce immune inflammatory suppression in healthy people, this effect is not robust enough to change inflammatory mediators' levels in situations of highly dysfunctional inflammatory activation. Nevertheless, the impact of cannabinoids in clinical outcomes of these conditions remains to be determined.
\end{abstract}

(c) 2020 S. Karger AG, Basel

\section{Introduction}

Cannabis sativa, also known as marijuana, hashish, or skunk, was used once in life by $3.9 \%$ of the world population according to the World Drug Report [1]. On the American continent, the USA was the country that registered the highest prevalence of lifetime use, with $11.6 \%$ of the population having used cannabisat least once in life.

Although these data indicate the use of cannabis worldwide is expressive, the literature about medical properties of cannabis is inconclusive and lacks literature reviews. Schlicker [2] revealed that cannabis derivatives, such as delta-9-tetrahydrocanabinol (delta-9-THC) and cannabidiol (CBD), are involved in several neurotransmitters systems, such as glutamatergic, serotonergic, noradrenergic, and dopaminergic systems, which are responsible for the therapeutic and recreational effects of cannabis.

Among the cannabis extracts, the THC is the main psychoactive component due to the lipophilic structure that allows the molecule to cross the blood-brain barrier [3]. Once in the central nervous system, the THC acts as a cannabinoid agonist, and its modulation of cannabinoid receptor 1 (CB1) is linked to pleasurable sensations. Such sensations are achieved because the CB1 is heterogeneously distributed around the brain, modulating the

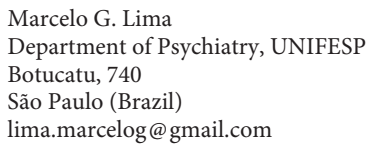


dopaminergic transmission in the limbic cortex and the association cortices [4]. The most commonly reported pleasure sensations are feeling of well being, calmness, relaxation, and hilarity [5]. Furthermore, according to Carlini et al. [5], the most common physical symptoms are xerostomia, red eyes, polyphagia, and tachycardia. Medical properties of cannabis extracts, however, are due to the several neurotransmitters involved on $\mathrm{CB} 1$ and CB2 (cannabinoid receptor 2) receptors [6].

The correlation between immune response and cannabis use has been explored, as in the longitudinal study performed by Kagen et al. [7], which aimed to evaluate the role of cannabis use on inducing sensitization to Aspergillus. It was important to find that cannabis users had a higher risk of fungal exposure and infection, increasing the variety of immunologic lung disorders presented by the subjects. Roth et al. [8] performed a study aiming to analyze the production of nitric oxide (NO) on cannabis users and the role of NO as an antimicrobial agent. The study provides the role of cannabis use decreasing $\mathrm{NO}$ production, which acts as an important mediator of antibacterial effects. So, these studies illustrate direct and indirect impact of cannabis use on the susceptibility to infections.

Within the medical effects of cannabis use, the antiinflammatory properties can be explored therapeutically. Klein et al. [9] explored the alteration of immune mediators referring the suppression of tumor necrosis factor alpha (TNF- $\alpha$ ) and other cytokines such as granulocytemacrophage colony-stimulating factor (GM-CSF), interleukin 6 (IL-6), interferon-gamma (IFN- $\gamma$ ), and interleukin 12 (IL-12) have also been observed following exposure to high affinity and psychoactive ligands such as cannabinoids and THC. MacCallum et al. [10] portray that cannabis extracts have important pharmacological properties, whereas THC has been noted to produce antiinflammatory effects by the antagonism of TNF- $\alpha$ [11] and to be a strong anti-emetic [12] and was recently demonstrated to be an agonist of the peroxisome proliferatoractivated receptor gamma (PPAR- $\gamma$ ) nuclear receptor with neuroprotective effects [13], as well as anticonvulsant efficacy [14]. CBD is also a powerful anti-emetic [15] and anti-anxiety agent [16] in rodents.

Koppel et al. [17] published a systematic review analyzing publications between 1948 and 2003 about the use of CBD on the treatment of multiple sclerosis (MS) and chronic pain, which registered the efficacy of oral extracts from cannabis that used combinations of THC/CBD or CBD only. Furthermore, a study conducted by Klein [18] found an anti-inflammatory potential for the cannabi- noids, which would be helpful in treatment of the inflammatory diseases, such as rheumatoid arthritis, lupus erythematosus, and MS.

Responding to potential aggressions, the immune system activates the innate and adaptive immunities by producing inflammatory cytokines, which mediate and potentiate the inflammatory process [19] that, in turn, signals an alteration of homeostasis. Although the immune system in normal conditions addresses potential aggressions and pathogen antigens, several dysfunctions turn the system to recognize the autoantigens as an aggressor and cause autoimmune diseases. Detailed information about immune cytokines, interleukins, and inflammatory cell function is presented in Table 1.

The cannabis extracts are capable of modulating immune function [20]. This modulation occurs because these extracts serve as secondary modulators. When mobilized coincidently with or shortly after first-line immune modulators such as lymphokines, they increase or decrease immune activity [18]. Furthermore, the CB2 is present in the immune system cells, thereby regulating cell migration and cytokine release [21]. These are the underlying mechanisms of the immunomodulatory effect of cannabinoids.

In conclusion, the cannabis extracts are recognized by their hallucinogens and therapeutic properties, but the medical literature about their use is inconclusive and even contradictory. We conducted a narrative review approaching the inflammatory markers in individuals who used cannabis aiming at supplying the lack of literature exploring the therapeutics effects of cannabis use and looking at the cannabinoids as a potential anti-inflammatory agent in humans.

\section{Materials and Methods}

We screened all articles that cited inflammatory mediators and any information about cannabis use. We searched the PubMed database for all articles published from 1980 to May 2019. We also examined the reference lists of these articles and retrieved studies that appeared to fulfill our criteria. Detailed information about the review numbers can be seen in Figure 1. Our search strategy included the mesh terms (inflammation OR immunity OR cytokines) AND cannabis, in order for the search to be as broad as possible. Also, we conducted an evidence level analysis according to the Oxford Centre for Evidence-Based Medicine - Levels of Evidence [22].

\section{Search Criteria}

The following inclusion criteria were used: (i) studies in humans and (iii) studies assessing inflammatory markers' serum levels in cannabis users and nonusers. 
Table 1. Inflammatory mediators and biological function according to Abbas et al. [21]

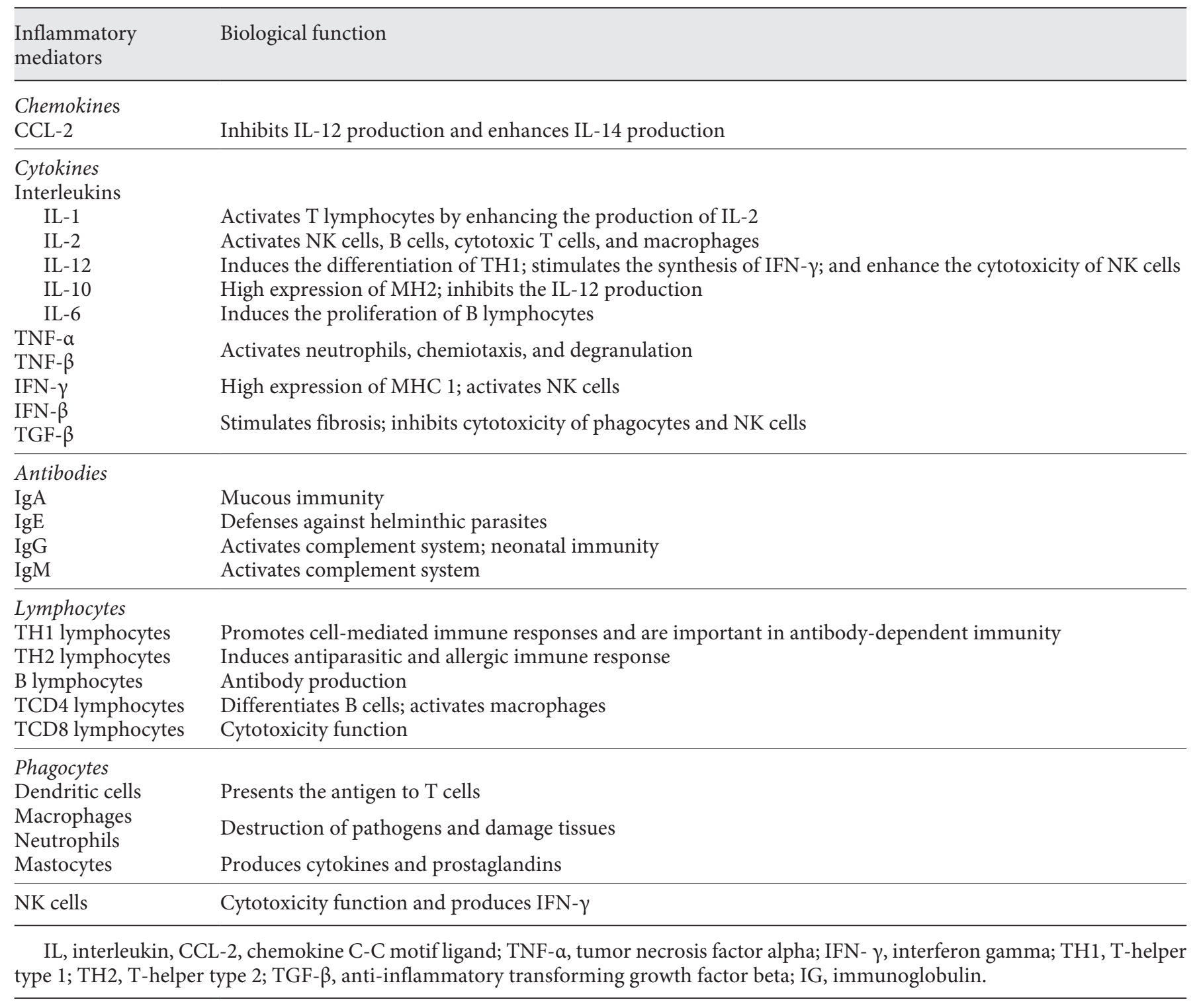

\section{Data Extraction}

Data were independently extracted by the first author (M.G.L.) using a structured form and reviewed by the senior author (T.M.F.). The following variables were extracted: (1) author's name, (2) year of publication, (3) country where the study was conducted, (4) sample size, (5) sample characteristics, (6) study design, (7) age range of the sample, (8) statistical analysis performed, (9) instrument(s) utilized, (10) exposure covariates, (11) outcome measures, (12) major findings, and (13) study limitations. Discrepancies were resolved by consensus, and a third author (E.B.) was consulted when needed. Data concerning cannabis use and its relationship with inflammatory markers were de- scribed, when available. It was not possible to describe or standardize cannabinoid consumption for all papers reviewed because many of them did not mention smoke patterns and also because articles applied very heterogeneous measurement methods. Publication including only cannabidiol or synthetic cannabinoids was not included.

\section{Data Analysis}

We described the results qualitatively because there was no quantitative information available to perform a meta-analysis. 


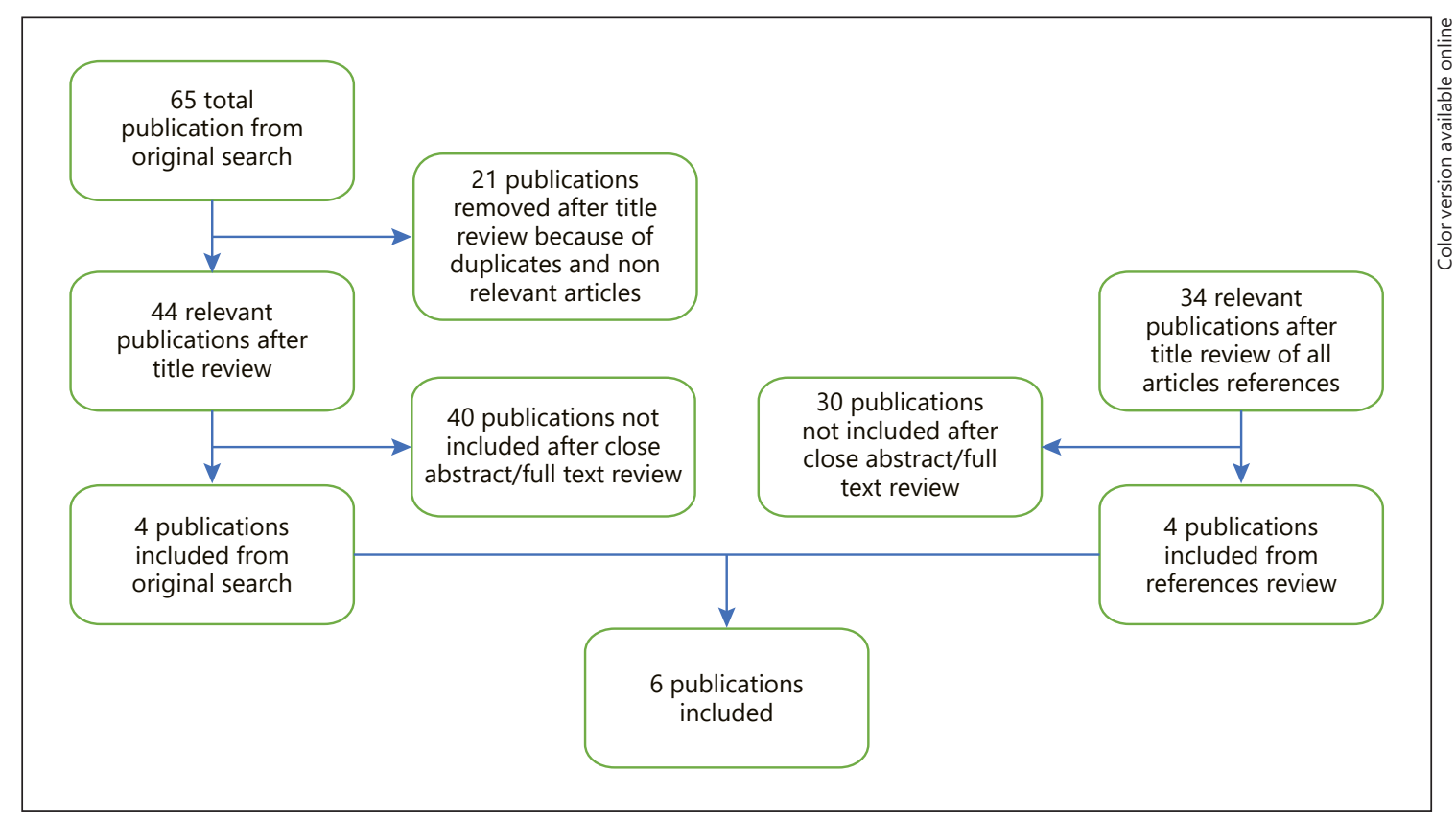

Fig. 1. Flowchart about review numbers.

\section{Results}

We identified 65 articles in our preliminary search. Of these references, 21 were excluded on the first analysis because of duplicates and nonrelevant articles. All 44 remaining articles were further reviewed, and 40 of these were excluded because they did not fulfill the inclusion criteria. The remaining 4 articles met our inclusion criteria and were fully reviewed, and data were extracted from them. Also, we identified 34 relevant publications after title review of all article's references in which only 2 publications met our inclusion criteria and were fully reviewed, and data were extracted from them.

Five out of the 6 papers in our review were longitudinal studies. In 4 of the papers, cannabis or its extracts were found to be negatively associated with immunity status, indicating that the higher the cannabis consumption, the lower the immunity cytokine levels went. The remaining 2 articles showed no association between cannabis use and serum immunity cytokine levels. The most commonly reported exposure covariates were age, sex, cannabis use, alcohol use, and tobacco use.

The results will be thoroughly presented in 3 subcategories: studies conducted among (i) healthy volunteers; (ii) cannabis users; and (iii) medical cannabis use in volunteers with general medical conditions. We considered that these categories should be analyzed separately as the clinical and medical profiles of participants should vary across them.

\section{Healthy Volunteers}

We found 2 studies conducted in healthy volunteers' samples, conducted by the same research team, Pacifi et al. [23, 24], in 2003 [23] and 2006 [24]. Both were longitudinal observational studies. On the 2003 article [23], 61 volunteers were included and 3 distinct groups were analyzed: polydrug users, cannabis users, and a control group with no drug use. The aim was to compare the cell-mediated immune response and cytokine release in cannabis users in relation to the control group. The major finding was that cannabis users had lower function on immune response, with a considerable decrease in inflammatory cytokine serum levels. The authors state that the small sample size might have been a limitation of that study. On the 2006 article [24], 94 volunteers were included and divided into the same 3 groups of the 2003 article [23]. The article analyzed the cell-mediated immune function and the occurrence of mild infectious diseases. It reported 3 important findings: (i) polydrug users had a big decrease in immune response and a considerable increase in antiinflammatory transforming growth factor $\beta 1$; therefore, (ii) polydrug users had an increase in mild common infections; finally, (iii) cannabis users had an intermediate decrease in immune response in relation to the control 


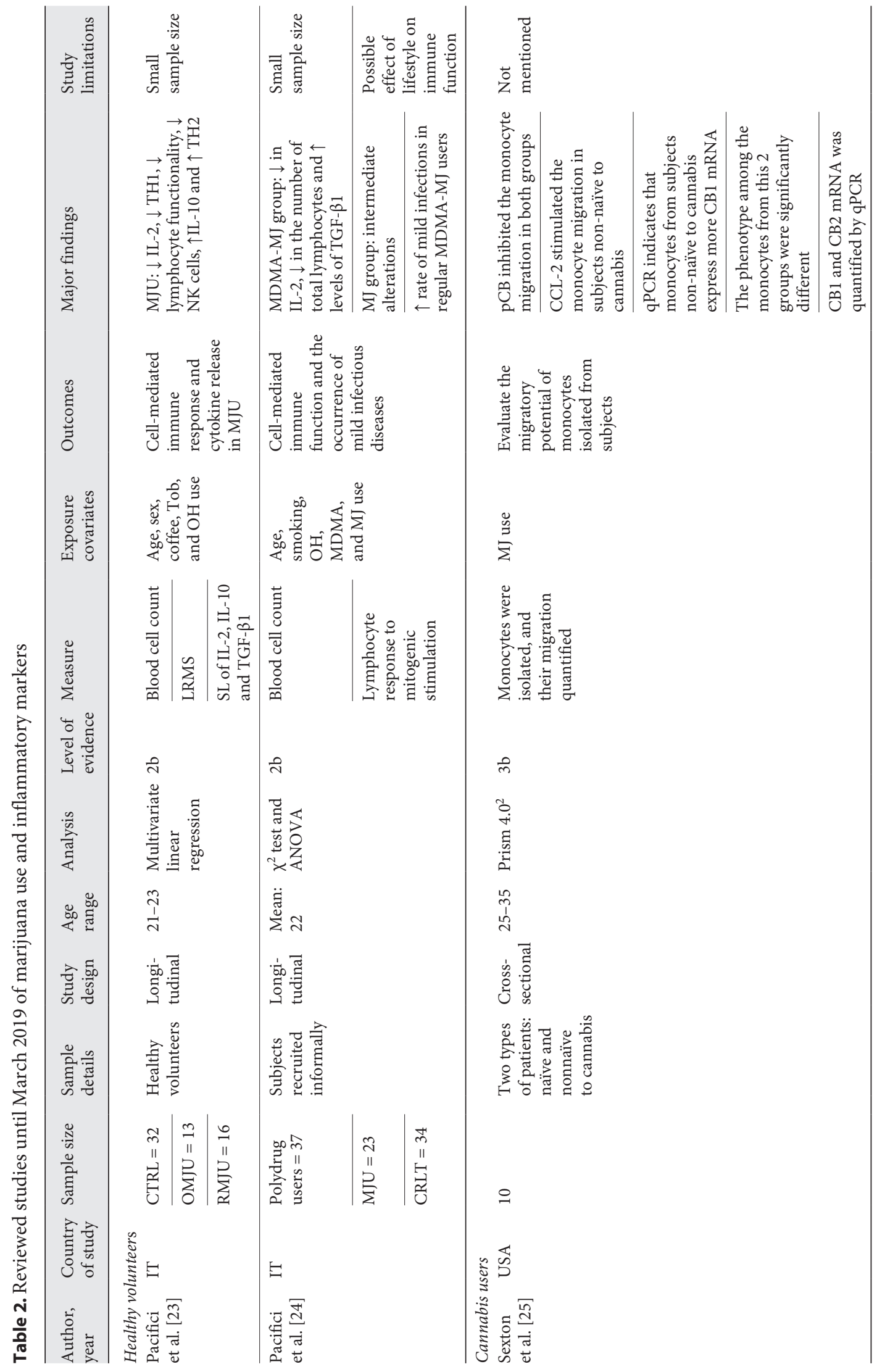




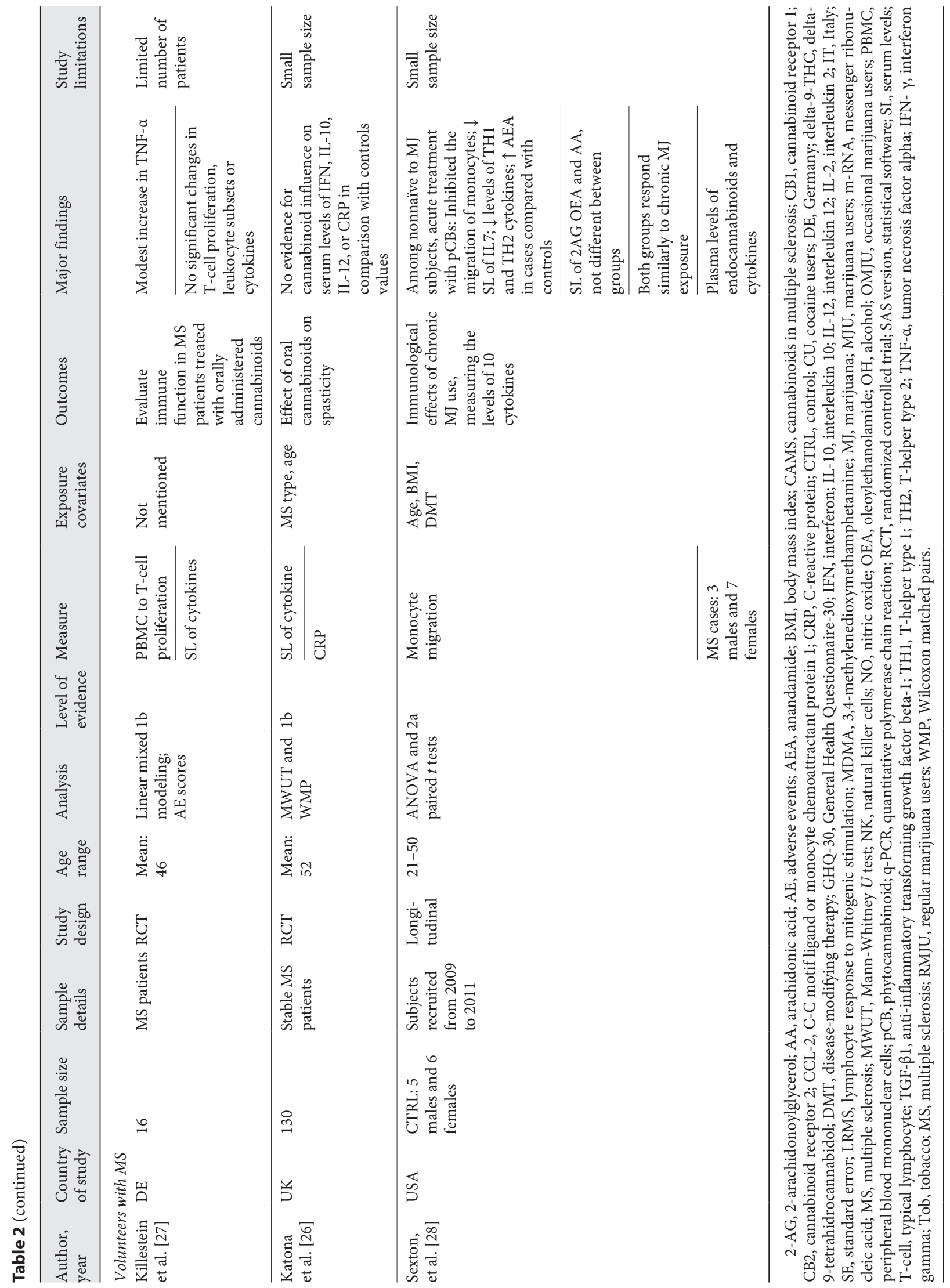


Table 3. Reviewed studies categorized by the Oxford Centre for Evidence-Based Medicine - Levels of Evidence in the order from highest to lowest levels A, B, C, and D

\begin{tabular}{lllll}
\hline & \multicolumn{2}{c}{ Evidence level } & & \\
\cline { 2 - 4 } & A & B & C & D \\
\hline Articles & Killestein et al. [29] & $\begin{array}{l}\text { Pacifici et al. [25] } \\
\text { Pacifici et al. [26] }\end{array}$ & \\
\cline { 2 - 4 } & Katona et al. [28] & Sexton et al. [27] & \\
& Sexton et al. [30] & \\
\hline
\end{tabular}

group. This article also had the small sample size limitation, and it did not consider the possible effect of lifestyle on immune function. Detailed information is presented in Table 2.

\section{Cannabis Users}

We found one article evaluating cannabis users, which was produced in the USA. Detailed information is presented in Table 2.

Sexton et al. [25] performed a cross-sectional study, evaluating 10 cannabis users. The aim of this study was to evaluate the migratory potential of isolated monocytes from cannabis users. It found that cannabinoids inhibited the migration of monocytes in both groups (naïve and nonnaïve to cannabis), and the monocytes from subjects nonnaïve to cannabis expressed more $\mathrm{CB} 1$ messenger ribonucleic acid (mRNA). Although the authors report no limitations, we can infer that unknowing about the acute and long-term effects of phytocannabinoid (pCB) on human circulating monocytes limits the comprehension of the study findings.

\section{Medical Cannabis Used in Volunteers with MS}

We found 3 articles using volunteers with generalized medical conditions treated with medical cannabis extracts. All of them included patients with MS. Detailed information is presented in Table 2.

Katona et al. [26] performed an RCT in the UK with 130 volunteers. The aim of that paper was to evaluate the effect of oral cannabinoids on spasticity. Patients with stable MS received dronabinol, Cannador ${ }^{\circledR}$, and matching placebo. Doses were adjusted according to side effects, with a maximal oral dose of $0.25 \mathrm{mg} / \mathrm{kg} /$ day of delta-9-THC. The study found no evidence of cannabinoid influence on serum levels of IFN, IL-10, IL-12, or CRP in comparison to control values. The small sample size is a limitation to this study.
Killestein et al. [27] performed a randomized controlled trial (RCT) in Germany with 16 volunteers: 10 had secondary progressive MS and 6, primary progressive MS. The aim of this paper was to evaluate immune function in MS patients treated with orally administered cannabinoids. This 2-fold study treated their volunteers with identical-appearing capsules containing dronabinol, Cannabis sativa plant extract or placebo. Volunteers were treated with 84 capsules for 4 weeks each. The study found modest increase in TNF- $\alpha$ and no significant changes in T-cell proliferation, leukocyte subsets, or cytokines. The small sample size is also a limitation to this study.

Finally, the third study was performed by Sexton et al. [28]. It was a longitudinal study aiming to describe the immunological effects of chronic cannabis use among MS patients, measuring the levels of 10 cytokines. It found that cannabis users showed inhibited monocyte migrations, decrease in serum IL-7, decrease in levels of lymphocyte Thelper type 1 (TH1) and lymphocyte T-helper type 2 (TH2) cytokines, and increase in anandamide (AEA) in comparison to controls. Small sample size was also a limitation.

\section{Highest Evidence}

A categorization of the included studies in accordance with Oxford Centre for Evidence-Based Medicine - Levels of Evidence criteria is presented in Table 3. Among "Healthy Volunteers," both studies, Pacifici et al. [23] and Pacifici et al.[24], were at the same level of evidence: $2 b$. Both studies are prospective cohorts without homogeneity with a longitudinal approach. Among "Marijuana Users," the only study, Sexton et al. [25] is classified as 3b. It is a case-control study with a small sample size performed on cross-sectional design. Among "Volunteers with Multiple Sclerosis," Katona et al. [26] and Killestein et al. [27] are classified as $1 \mathrm{~b}$. That because the studies are an RCT performed with a homogeneous population with a longitudinal approach.

\section{Discussion}

The aim of this study was to review the literature about the effect of cannabis use on inflammatory markers. The main findings were as follows: (i) among healthy volunteers and among cannabis users, cannabinoids seemed to decrease the inflammatory response, thus decreasing the immune response leading in turn to a higher risk of infections; (ii) among patients with MS, cannabinoids seemed to have little impact on the inflammatory markers' levels. 
A review published in 2005 analyzed the immune system impacts of the cannabis extracts [29]. They demonstrated an apparent contradiction about the immune reactions; although the majority of studies showed that the administration of cannabinoids has inhibitory effects on immune cells, a number of recent studies have demonstrated that the endocannabinoids may have some stimulatory impact on the immune system. This apparent contradiction may be due to a biphasic response related to the cannabinoid ligand concentration as many of the inhibitory effects of cannabinoids in vitro are in the micromolar concentration range, whereas stimulatory concentrations are in the nanomolar range. In spite of that, the articles analyzed in this study, which used only human volunteers, reveal that among healthy volunteers and cannabis users, exogenous cannabinoids seemed to decrease the inflammatory response, thus decreasing immune response and leading to a higher risk of infections. Thus, on in vivo research, the contradiction about the cannabis use on inflammatory effects is smaller than that on in vitro experiments [29].

MS is an autoimmune inflammatory disease with several physical and mental symptoms, which affects deeply the patient's quality of life [30-33]. These MS-associated symptoms can be treated by current drug therapies that cause considerable side effects, including hallucinations, hypotension, seizures, anxiety, weakness, and nausea [34]. According to Goodin et al. [34], the effectiveness of the disease-modifying therapeutics agents in reducing disability progression in relapsing-remitting MS patients is unclear. The articles analyzed in this study, on the contrary, reveal that cannabis extracts and cannabinoids promote improvement in MS symptoms and seemed to have little impact on the serum inflammatory markers' levels $[26,27]$, which suggests that improvements may occur through different mechanisms involving the cannabinoids. Considering that immune diseases, such as MS, systemic lupus erythematosus, and rheumatoid arthritis are considerably disabling both physically and mentally, the potential of decrease in the immune function caused by cannabis extracts and cannabinoids could provide a pathway through which inflammatory diseases could be addressed by reducing disease immune activity.

These findings must be interpreted in light of some limitations. One of them is that we included only publications in English and Portuguese. Although this is a limitation, a previous study has stated that language restriction does not usually alter the main findings of systematic reviews [35]. Moreover, this review focused only on human studies, and the percentage of the $\mathrm{CBD} / \mathrm{THC}$ relation in each study was not provided. This $\mathrm{CBD} / \mathrm{THC}$ relation is important because the THC-rich compounds have serious limitations such as unpredictable gastrointestinal absorption and potential intoxication and disorientating central nervous system effects at the higher doses [36]. The addition of CBD to THC should ameliorate the intoxicating effects of THC, paranoia, and euphoria associated with THC, with diminished potential for abuse [37].

About the limitations of the reviewed articles, with regards to sample size, only one article had a sample size larger than 100 individuals. We can also mention heterogeneity and lack of standardization of the articles regarding the statistical analyses used in each study.

\section{Statement of Ethics}

Considering the secondary pattern of reviews, the ethical approval was not required because all articles reviewed were approved by their corresponding ethics committees.

\section{Conflict of Interest Statement}

The authors have no conflicts of interest to declare.

\section{Funding Sources}

This study was funded by FAPESP (Fundacao de Apoio a Pesquisa do Estado de Sao Paulo) under research grant protocol: 2019/13088-0.

\section{Author Contributions}

M.G.L., T.M.F., and E.B. were responsible for the study concept and design. M.G.L. was responsible for data extraction and summarization of findings. V.S.T. and T.M.F. supervised data extraction and confection of the tables. V.S.T., E.B., and T.M.B. assisted with data analysis and interpretation of findings. M.G.L. drafted the manuscript. V.S.T., E.B., and T.M.F. provided critical revision of the manuscript for important intellectual content. All authors critically reviewed the content and approved the final version for publication.

\section{References}

1 United Nations Office of Drugs and Crime. World Drug Report 2018. Nations Publication. Sales No. E.18.XI.9.

2 Schlicker E, Kathmann M. Modulation of transmitter release via presynaptic cannabinoid receptors. Trends Pharmacol Sci. 2001 Nov;22(11):565-72.

3 Mechoulam R. Marihuana chemistry. Science. 1970;168(3936):1159-66. 
4 Biegon A, Kerman IA. Autoradiographic study of pre- and postnatal distribution of cannabinoid receptors in human brain. Neuroimage. $2001 ; 14(6)$ : 1463-8.

5 Carlini EA, Nappo SA, Galduróz JCF, Noto AR. Drogas psicóticas: o que são e como agem. Revista IMESC no. 3. 2001;9-35.

6 Ribeiro JAC, Machado JQ. A Cannabis e suas aplicações terapêuticas. Porto: Universidade Fernando Pessoa- Faculdade de Ciências da Saúde; 2014.

7 Kagen SL, Kurup VP, Sohnle PG, Fink JN. Marijuana smoking and fungal sensitization. J Allergy Clin Immunol. 1983 Apr;71(4):38993.

8 Roth MD, Whittaker K, Salehi K, Tashkin DP, Baldwin GC. Mechanisms for impaired effector function in alveolar macrophages from marijuana and cocaine smokers. J Neuroimmunol. $2004 \mathrm{Feb} ; 147(1-2): 82-6$.

9 Klein TW, Lane B;Newton CA, Friedman H. The cannabinoid system and cytokine network. Proc Soc Exp Biol Med. 2000 Oct: 225(1):1-8.

10 MacCallum CA, Russo EB. Practical considerations in medical cannabis administration and dosing. Eur J Intern Med. 2018;49:12-9.

11 Verhoeckx KC, Korthout HA, van MeeterenKreikamp AP, Ehlert KA, Wang M, van der Greef J, et al. Unheated cannabis sativa extracts and its major compound THC-acid have potential immuno-modulating properties not mediated by CB1 and CB2 receptor coupled pathways. Int Immunopharmacol. 2006;6(4):656-65.

12 Rock EM, Sticht MA, Parker LA. Effects of phytocannabinoids on nausea and vomiting. In: Pertwee RG, editor. Handbook of cannabis. Oxford, UK: Oxford University Press; 2014. p. 435-54.

13 Nadal X, Del Río C, Casano S, Palomares B, Ferreiro-Vera C, Navarrete C, et al. Tetrahydrocannabinolic acid is a potent PPAR $\gamma$ agonist with neuroprotective activity. Br J Pharmacol. 2017;174(23):4263-76.

14 Sulak D, Saneto R, Goldstein B. The current status of artisanal Cannabis for the treatment of epilepsy in the United States. Epilepsy Behav. 2017;70(Pt B):328-33.

15 Bolognini D, Rock EM, Cluny NL, Cascio MG, Limebeer CL, Duncan M, et al. Cannabidiolic acid prevents vomiting in Suncus murinus and nausea-induced behaviour in rats by enhancing 5-HT1A receptor activation. $\mathrm{Br}$ J Pharmacol. 2013;168(6):1456-70.

16 Rock EM, Limebeer CL, Petrie GN, Williams LA, Mechoulam R, Parker LA. Effect of prior foot shock stress and $\Delta$. Psychopharmacology. 2017;234:2207-17.

17 Koppel B, Brust J, Fife T, Bronstein J, Youssof S, Gronseth G, et al. Systematic review: efficacy and safety of medical marijuana in selected neurologic disorders: report of the Guideline Development Subcommittee of the American Academy of Neurology. Neurology. 2014;82(17):1556-63.

18 Klein TW. Cannabinoid-based drugs as antiinflammatory therapeutics. Nat Rev Immunol; 2005. p. 400-411. https://www.nature. com/articles/nri1602.

19 Abbas AK, Lichtman AH, Pillai S. Imunologia celular e molecular. 8th ed. Rio de Janeiro: Elsevier; 2015.

20 Eisenstein TK. Effects of cannabinoids on Tcell function and resistance to infection. J Neuroimmune Pharmacol. 2015 Jun;10(2): 204-16.

21 Contino M, Capparelli E, Colabufo NA, Bush AI. Editorial: the CB2 cannabinoid system: a new strategy in neurodegenerative disorder and neuroinflammation. Front. Neurosci. 2017; 11:196

22 Phillips B, Ball C, Sackett D, Badenoch D, Straus S, Haynes B, et al. CEBM- Levels of evidence (March 2009). Oxford Centre for Evidence-Based Medicine. 2009. https://www. cebm.net/2009/06/oxford-centre-evidenceb ased-medicine-levels-evidencemarch-2009/.

23 Pacifici R, Zuccaro P, Pichini S, Roset PN, Poudevida S, Farré M, et al. Modulation of the immune system in cannabis users. JAMA. 2003 Apr 16;289(15):1929-31.

24 Pacifici R, Zuccaro P, Farré M, Poudevida S, Abanades S, Pichini S, et al. Combined immunomodulating properties of 3,4 methylenedioxymethamphetamine (MDMA) and cannabis in humans. Addiction. 2007 Jun;102(6): 931-6.

25 Sexton M, Silvestroni A, Möller T, Stella N. Differential migratory properties of monocytes isolated from human subjects naïve and non-naïve to Cannabis. Inflammopharmacology. 2013 Jun;21(3):253-9.

26 Katona S, Kaminski E, Sanders H, Zajicek J. Cannabinoid influence on cytokine profile in multiple sclerosis. Clin Exp Immunol. 2005 Jun;140(3):580-5.

27 Killestein J, Hoogervorst EL, Reif M, Blauw B, Smits M, Uitdehaag BM, et al. Immunomodulatory effects of orally administered cannabinoids in multiple sclerosis. J Neuroimmunol. 2003 Apr; 137(1-2): 140-3.

28 Sexton M, Cudaback E, Abdullah RA, Finnell J, Mischley LK, Rozga M, et al. Cannabis use by individuals with multiple sclerosis: effects on specific immune parameters. Inflammopharmacology. 2014 Oct;22(5):295-303.

29 Croxford JL, Yamamura T. Cannabinoids and the immune system: potential for the treatment of inflammatory diseases? J Neuroimmunol. 2005 Sep;166(1-2):3-18.

30 Alonso R, Contenttini EC, Imhoff G, Lopez PA, Rubestein A, Tizio S. Barriers against a successful MS treatment: the importance of effectiveness beyond efficacy. Mult Scler Relat Disord. 2019;30:129-35.

31 Patrick S, Connick P. Psychometric properties of the PHQ-9 in multiple sclerosis: a systematic review. PLoS One. 2019 Feb 19;14(2): e0197943.

32 Pugliatti M, Rosati G, Carton H, Riise T, Drulovic J, Vécsei L, et al. The epidemiology of multiple sclerosis in Europe. Eur J Neurol. 2006;13(7):700-22.

33 Zwibel HL, Smrtka J. Improving quality of life in multiple sclerosis: an unmet need. Am J Manag Care. 2011;17(Suppl 5):S139-45.

34 Goodin DS, Frohman EM, Garmany GP Jr, Halper J, Likosky WH, Lublin FD, et al. Disease modifying therapies in multiple sclerosis: report of the Therapeutics and Technology Assessment Subcommittee of the American Academy of Neurology and the MS Council for Clinical Practice Guidelines. Neurology. 2002 Jan 22;58(2):169-78.

35 Morrison A, Polisena J, Husereau D, Moulton K, Clark M, Fiander M, et al. The effect of English-language restriction on systematic review-based meta-analyses: a systematic review of empirical studies. Int J Technol Assess Health Care. 2012 Apr;28(2):138-44.

36 Kramer JL. Medical marijuana for cancer. CA Cancer J Clin. 2015;65(2):109-22.

37 Allsop DJ, Copeland J, Lintzeris N, Dunlop AJ, Montebello M, Sadler C, et al. Nabiximols as an agonist replacement therapy during cannabis withdrawal: a randomized clinical trial. JAMA Psychiatry. 2014;71(3):281-91. 